\title{
Prevalence of fosfomycin resistance and gene mutations in clinical isolates of methicillin-resistant Staphylococcus aureus
}

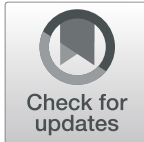

Yi-Chien Lee ${ }^{1,2}$, Pao-Yu Chen ${ }^{3}$, Jann-Tay Wang ${ }^{3,4}$ and Shan-Chwen Chang ${ }^{3 *}$

\begin{abstract}
Background: Fosfomycin exhibits excellent in vitro activity against multidrug-resistant pathogens, including methicillin-resistant Staphylococcus aureus (MRSA). Increasing fosfomycin resistance among clinical MRSA isolates was reported previously, but little is known about the relative abundance of Fosfomycin resistance genes in MRSA isolates circulating in Taiwan.
\end{abstract}

Methods: All MRSA isolates, collected in 2002 and 2012 by the Taiwan Surveillance of Antimicrobial Resistance (TSAR) program, were used in this study. Susceptibility to various antimicrobial agents, including fosfomycin, was determined by broth microdilution. Genetic determinants of fosfomycin resistance, including fos $B$ carriage and murA, glpT and uhpT mutations, were investigated using PCR and sequencing of amplicons. Staphylococcal protein A (spa) typing was also performed to determine the genetic relatedness of MRSA isolates.

Results: A total of 969 MRSA strains, 495 in the year 2002 and 474 in the year 2012, were analyzed. The overall in vitro susceptibility was $8.2 \%$ to erythromycin, $18.0 \%$ to clindamycin, $29.0 \%$ to tetracycline, $44.6 \%$ to ciprofloxacin, $57.5 \%$ to trimethoprim/sulfamethoxazole, $86.9 \%$ to rifampicin, $92.9 \%$ to fosfomycin and $100 \%$ to linezolid and vancomycin. A significant increase in the fosfomycin resistance rate was observed from $3.4 \%$ in 2002 to $11.0 \%$ in 2012. Of 68 fosfomycin-resistant MRSA isolates, several genetic backgrounds probably contributing to fosfomycin resistance were identified. Twelve isolates harbored the fos $B$ gene, and various mutations in murA, uhpT, and glpT genes were noted in 11, 59, and 66 isolates, respectively. The most prevalent gene mutations were found in the combination of uhpT and glpT genes (58 isolates). The vast majority of the fosfomycin-resistant MRSA isolates belonged to spa type t002.

Conclusions: An increased fosfomycin resistance rate of MRSA isolates was observed in our present study, mostly due to mutations in the glpT and uhpT genes. Clonal spread probably contributed to the increased fosfomycin resistance.

Keywords: Fosfomycin, Resistance, Gene mutations, Methicillin-resistant Staphylococcus aureus

\footnotetext{
*Correspondence: changsc@ntu.edu.tw

${ }^{3}$ Department of Internal Medicine, National Taiwan University Hospital, 7

Chung-Shan South Road, 100 Taipei, Taiwan

Full list of author information is available at the end of the article
}

(C) The Author(s). 2020 Open Access This article is licensed under a Creative Commons Attribution 4.0 International License, which permits use, sharing, adaptation, distribution and reproduction in any medium or format, as long as you give appropriate credit to the original author(s) and the source, provide a link to the Creative Commons licence, and indicate if changes were made. The images or other third party material in this article are included in the article's Creative Commons licence, unless indicated otherwise in a credit line to the material. If material is not included in the article's Creative Commons licence and your intended use is not permitted by statutory regulation or exceeds the permitted use, you will need to obtain permission directly from the copyright holder. To view a copy of this licence, visit http://creativecommons.org/licenses/by/4.0/ The Creative Commons Public Domain Dedication waiver (http://creativecommons.org/publicdomain/zero/1.0/) applies to the data made available in this article, unless otherwise stated in a credit line to the data. 


\section{Background}

Fosfomycin, a phosphonic acid derivative produced by Streptomyces spp. and discovered in 1969 [1], displays broad-spectrum activity against both Gram-negative and Gram-positive pathogens. It is a bactericidal antimicrobial agent that interferes with the enzyme-catalyzed bacterial cell wall synthesis [2]. Numerous studies have demonstrated the excellent in vitro susceptibility of multidrug-resistant and extensively drug-resistant organisms (MDRO and XDROs) to fosfomycin, including vancomycin-resistant enterococci (VRE) (96\%) [3], ESBL-producing Enterobacteriaceae (87.7\%) [4], carbapenem-resistant Gram-negative bacteria (99\%) [5], and methicillin-resistant Staphylococcus aureus (MRSA) (99.6\%) [6]. Additionally, the synergistic effect of fosfomycin in combination with other relevant antibiotics against the above-mentioned MDR microorganisms, evaluated by time-kill experiments, checkerboard analysis and E-test methods [7-9], was promising. These studies indicated that fosfomycin could be a potential treatment option for the difficult-to-treat infections caused by drug-resistant organisms.

Among MDROs, MRSA is a major human pathogen which causes various dangerous infections, such as bacteremia, endocarditis, and abscess, in both community and hospital settings [10]. Fosfomycin alone or combined with other antimicrobial agents exhibited favorable in vitro activity against MRSA $[6,8,11,12]$, and more than $70 \%$ clinical cure was observed with fosfomycin administration for the treatment of MRSA infection [13, 14]. However, S. aureus with fosfomycin resistance developed and rose rapidly by $30-70 \%$ in China [15]. The mechanism of bacterial resistance to fosfomycin could involve either a chromosomeassociated defective transport system or plasmidmediated fosfomycin-inactivating enzymes. First, two key transporter systems, GlpT and UhpT, mediated the entry of fosfomycin into bacterial cells [16]. Once mutations in the chromosomal $g l p T$ and $u h p T$ genes occurred, reduction in permeability with subsequent conferred MRSA resistance to fosfomycin was observed [16-18]. Second, the murA gene mutants exhibited lower affinity for fosfomycin [19], conferring various degrees of drug resistance. Moreover, a few fosfomycin-modifying enzymes, including FosA, FosB, FosC, and FosX, catalyzed the inactivation of fosfomycin [20-22], and only FosB is produced by Grampositive bacteria [23]. Most of the previous studies investigated the mechanism of fosfomycin resistance among Gram-negative bacteria, and only limited information about the resistance mechanism of Grampositive pathogens, particularly MRSA, is available. Hence, in the present study, we aimed to survey the prevalence of fosfomycin resistance and the associated
uhpT, glpT, murA, and fos $B$ genetics in clinical isolates of MRSA in Taiwan.

\section{Methods}

\section{Bacterial isolates}

All MRSA strains, collected in 2002 and 2012 through the TSAR program from different hospitals in Taiwan, were used in this study. The principles of isolate collection by the TSAR program had been described clearly in a previous study [8]. Duplicate isolates were excluded, and a total of 969 MRSA isolates, 495 collected in 2002 and 474 in 2012, were analyzed. All these strains were identified as $S$. aureus by performing Gram staining, a catalase-activity test, and a coagulase latex agglutination test (automated VITEK-2 system, Biomerieus, France). Methicillin resistance was ascertained using agar disk diffusion (Kirby-Bauer), according to the guidelines established by the Clinical and Laboratory Standards Institute (CLSI) [24]. The study was approved by the Ethical Committee of the National Taiwan University Hospital (NTUH-IRB No. 201504056RINB).

\section{Antimicrobial susceptibility}

The antimicrobial susceptibility to clindamycin, ciprofloxacin, erythromycin, linezolid, rifampicin, trimethoprim/sulfamethoxazole, tetracycline, fosfomycin, and vancomycin was determined using a broth microdilution method according to the CLSI recommendations [24], and the results were interpreted using the criteria for $S$. aureus provided by the CLSI [24]. Staphylococcus aureus ATCC 29213 was used as the internal control for each run of the susceptibility test.

\section{Genetic analysis}

DNA of 68 fosfomycin-resistant MRSA isolates was harvested using a DNA Extraction System kit (Viogene, New Taipei City, Taiwan) according to the manufacturer's instructions. The presence of $f o s B$ was detected by PCR using the previously described primers [25], and the full nucleotide sequence of three genes (murA, uhpT, and $g l p T$ ) was determined by combing direct sequencing and primer walking with the individual PCR products. Primers, used in the present study, are shown in Table 1. The PCR and sequencing procedures were described in prior studies $[15,25]$.

\section{Molecular typing}

Staphylococcal protein A (spa gene) typing was performed for 68 fosfomycin-resistant MRSA isolates. The highly variable $\mathrm{X}$ region in spa was amplified by PCR using the previously described primers [26]. The purified PCR products were sequenced, and the spa types were confirmed by analyzing the nucleotide sequence utilizing 
Table 1 PCR primers of fos $A$, fos $B$, fos $C$, murA, glpT, and uhpT genes

\begin{tabular}{|c|c|c|c|c|}
\hline Primers & Genes & Primer sequences $(5>3)$ & Product size & References \\
\hline fosB-F & fosB & CAGAGATATTITAGGGGCTGACA & $312 \mathrm{bp}$ & [25] \\
\hline fos $B-R$ & & СТCAATCTATСTTСТAAACTTCСТG & & \\
\hline murA-F & murA & GCCCTTGAAAGAATGGTTCGT & $1600 \mathrm{bp}^{\mathrm{a}}$ & NC_002745. $2^{\mathrm{b}}$ \\
\hline murA-R & & GTTACAATACTCGACGCAGGT & & \\
\hline glpT-F & glpT & TGAATAAAACAGCAGGGCAA & $1699 \mathrm{bp}^{\mathrm{a}}$ & NC_002745.2 $2^{\mathrm{b}}$ \\
\hline glpT-R & & CACAGCTAGTATGTATAACGAC & & \\
\hline uhpT-F & uhpT & TGTGTITATGTTCAGTATITTGGA & $1571 b p^{a}$ & NC_002745. $2^{\mathrm{b}}$ \\
\hline uhpT-R & & TCTTTCATCTCTTCACGCAC & & \\
\hline
\end{tabular}

a $P C R$ product including surrounding sequences adjacent to target gene

${ }^{b}$ GenBank-EMBL-DDBL accession number

BioNumerics Version 6.5 (Applied Maths NV, SintMartens-Latem, Belgium) [27].

\section{Statistical analysis}

Categorical variables were expressed as percentages, and Fisher's exact test with two-sided comparison was utilized for the assessment of statistical significance.

\section{Results}

\section{Susceptibilities to tested antimicrobial agents}

The overall susceptibilities to various antibiotics was demonstrated in Table 2. Susceptibility rates to clindamycin, erythromycin, tetracycline, and trimethoprim/sulfamethoxazole increased from 2002 to 2012; however, the susceptibility rates to rifampicin and fosfomycin between 2002 and 2012 decreased statistically significantly, shown in Table 2. All tested MRSA isolates remained 100\% susceptible to linezolid and vancomycin. Among the fosfomycinresistant MRSA isolates, lower susceptibility rates to clindamycin, ciprofloxacin, erythromycin, and rifampicin were noted compared with those of the fosfomycin-susceptible group. In contrast, the susceptibility rates to trimethoprim/ sulfamethoxazole and tetracycline within the fosfomycinsusceptible group was lower (Table 3).

Table 2 Antibiotics susceptibilities grouped by study year

\begin{tabular}{lllll}
\hline Antibiotics $^{\mathrm{a}}$ & $\begin{array}{l}\text { Overall } \\
\text { susceptibilities } \\
\end{array}$ & \multicolumn{3}{l}{ Susceptibilities by years (\%) } \\
\cline { 3 - 5 } Clindamycin & $174(18.0)$ & $49(9.9)$ & $125(26.4)$ & $<0.001$ \\
Ciprofloxacin & $432(44.6)$ & $224(45.3)$ & $208(44.0)$ & 0.699 \\
Erythromycin & $79(8.2)$ & $17(3.4)$ & $62(13.1)$ & $<0.001$ \\
Linezolid & $968(100)$ & $495(100)$ & $473(100)$ & - \\
Rifampicin & $841(86.9)$ & $456(92.1)$ & $385(81.4)$ & $<0.001$ \\
SXT & $557(57.5)$ & $236(47.7)$ & $321(67.9)$ & $<0.001$ \\
Tetracycline & $281(29.0)$ & $70(14.1)$ & $211(44.6)$ & $<0.001$ \\
Fosfomycin & $900(93.0)$ & $478(96.6)$ & $422(89.2)$ & $<0.001$ \\
Vancomycin & $968(100)$ & $495(100)$ & $473(100)$ & - \\
\hline
\end{tabular}

${ }^{a}$ Antibiotic abbreviation: SXT, trimethoprim/sulfamethoxazole
Prevalence of fosfomycin resistance genes

Patients with fosfomycin-resistant MRSA colonization/ infection have a median age of 79 years (interquartile range: 69-85), which is significantly elder than that of patients with fosfomycin-susceptible MRSA (median, 59; interquartile range: 33-76). Most of those fosfomycinresistant MRSA isolates were collected from the hospitals located in central (37 isolates) or southern (20 isolates) Taiwan, but the majorities of fosfomycinsusceptible MRSA strains were isolated from central (34.9\%) and northern Taiwan (28\%). Of 68 fosfomycinresistant MRSA isolates, 12 strains harbored the fos $B$ gene with fosfomycin MICs ranging from 128 to $>2048$ $\mathrm{mg} / \mathrm{L}$ (Table 4). Classification of different mutations in the murA, uhpT, and glpT genes, including various nucleic acid deletions and amino acid substitutions, was defined as following: type ImurA, G257D; type IImurA, D278E; type IIImurA, deletion at position 717; type IVmurA, G322S; type VmurA, L162I; type VImurA, E271Q; type VIImurA, G240R; type IuhpT, A279V; type IIuhpT, A297V/E225D; type IIIuhpT, F267L/L281X; type IVuhpT, G161R; type IglpT, A434V; type IIglpT, W147X; type IIIglpT, F197I; type IVglpT, A434V/ G399S; type VglpT, C57X; type VIglpT, T313K. Nevertheless, a total of 11 isolates expressed a murA mutation, and seven different subtypes, including type $\mathrm{I}_{\text {murA }}$ to type $\mathrm{VII}_{\text {murA }}$, were identified in these mutant genes. Only type $\mathrm{III}_{\text {murA }}$ led to a deletion of one nucleic acid at position 717 , and the other six mutations (type I- $\mathrm{II}_{\text {murA }}$ and type $\mathrm{IV}-\mathrm{VII}_{\text {murA }}$ ) resulted in distinct amino acid substitutions within the MurA protein. The most commonly encountered fosfomycin-resistant murA mutant was type I (4 isolates).

Sixty-six of 68 fosfomycin-resistant MRSA strains contained one of the six different mutations (type $\mathrm{I}^{-\mathrm{VI}_{\text {glp }} \text { ) }}$ found in the $g l p T$ gene with the majority containing a type $\mathrm{I}_{g l p T}$ mutation (60 isolates). Each of these mutations caused amino acid substitutions within the GlpT protein. Furthermore, four different mutations (type I$\mathrm{IV}_{u h p T}$ ) were recognized in the $u h p T$ gene of the 59 
Table 3 Susceptibilities of different antibiotics in methicillin-resistant Staphylococcus aureus isolates divided by sensitive to fosfomycin and resistance to fosfomycin

\begin{tabular}{llll}
\hline Antibiotics $^{a}$ & \multicolumn{2}{c}{ Susceptibilities by (\%) } & \multicolumn{1}{c}{$\begin{array}{c}\text { Statistics } \\
\text { Fosfomycin-Susceptible }(n=900)\end{array}$} \\
\hline Clindamycin & $173(19.2)$ & Fosfomycin-Resistant $(n=68)$ & $<0.001$ \\
Ciprofloxacin & $431(47.9)$ & $1(1.5)$ & $<0.001$ \\
Erythromycin & $79(8.8)$ & $1(1.5)$ & 0.005 \\
Linezolid & $900(100)$ & $6(0)$ & - \\
Rifampicin & $833(92.6)$ & $68(100)$ & $<0.001$ \\
SXT & $495(55.0)$ & $9(13.2)$ & $<0.001$ \\
Tetracycline & $221(24.6)$ & $62(91.2)$ & $<0.001$ \\
Vancomycin & $900(100)$ & $60(88.2)$ & - \\
\hline
\end{tabular}

${ }^{a}$ Antibiotic abbreviation: SXT, trimethoprim/sulfamethoxazole

fosfomycin-resistant MRSA isolates with type $\mathrm{I}_{u h p T}$ as the most prevalent (55 isolates). Similarly, the four mutations resulted in amino acid substitutions within the UhpT protein. Likewise, 58 fosfomycin-resistant MRSA isolates displayed dual resistance mechanisms. The details of those fosfomycin-resistant MRSA isolates harboring different types of mutation genes were described in Table 4.

\section{Molecular typing}

The 68 fosfomycin-resistant MRSA strains were classified into several spa types, including t002 (52 isolates), t037 (5 isolates), and other spa types. The 52 spa t002 fosfomycin-resistant isolates had a greater proportion (51/52) of high fosfomycin MICs (1024 > 2048) than in the other $16(12 / 16)$ fosfomycin-resistant isolates $(p=$ 0.019). Among them, 11 isolates harbored the $f o s B$ gene;

Table 4 Distributions of fosfomycin-resistant related genes corresponding MIC values among MRSA isolates

\begin{tabular}{|c|c|c|c|c|c|c|c|c|c|c|c|}
\hline \multirow{2}{*}{$\begin{array}{l}\text { uhpT } \\
\text { mutation }^{a}\end{array}$} & \multirow{2}{*}{$\begin{array}{l}\text { glpT } \\
\text { mutation }^{\mathrm{b}}\end{array}$} & \multirow{2}{*}{$\begin{array}{l}\text { murA } \\
\text { mutation }^{c}\end{array}$} & \multirow{2}{*}{$\begin{array}{l}\text { fos } B \\
\text { gene }\end{array}$} & \multicolumn{2}{|c|}{ t002 } & \multicolumn{2}{|c|}{ t037 } & \multicolumn{2}{|c|}{ Other spa types } & \multicolumn{2}{|c|}{ Total } \\
\hline & & & & $n$ & FOS range & $n$ & FOS range & $n$ & FOS range & $n$ & FOS range \\
\hline Wild type & Wild type & Wild type & Positive & 0 & - & 0 & - & 1 & 128 & 1 & 128 \\
\hline Wild type & Type I & Wild type & Positive & 1 & $>2048$ & 0 & - & 0 & - & 1 & $>2048$ \\
\hline Wild type & Type I & Wild type & Negative & 3 & $>2048$ & 0 & - & 1 & $>2048$ & 4 & $>2048$ \\
\hline Wild type & Type II & Type I & Negative & 0 & - & 2 & $>2048$ & 0 & - & 2 & $>2048$ \\
\hline Wild type & Type VI & Wild type & Negative & 0 & - & 1 & 64 & 0 & - & 1 & 64 \\
\hline Type I & Wild type & Type I & Negative & 0 & - & 1 & 128 & 0 & - & 1 & 128 \\
\hline Type I & Type I & Wild type & Positive & 9 & $>2048$ & 0 & - & 0 & - & 9 & $>2048$ \\
\hline Type I & Type I & Wild type & Negative & 31 & $1024->2048$ & 0 & - & 7 & $>2048$ & 38 & $1024->2048$ \\
\hline Type I & Type I & Type III & Positive & 1 & $>2048$ & 0 & - & 0 & - & 1 & $>2048$ \\
\hline Type I & Type I & Type IV & Negative & 2 & $>2048$ & 0 & - & 0 & - & 2 & $>2048$ \\
\hline Type I & Type I & Type V & Negative & 1 & $>2048$ & 0 & - & 0 & - & 1 & $>2048$ \\
\hline Type I & Type I & Type VII & Negative & 1 & $>2048$ & 0 & - & 0 & - & 1 & $>2048$ \\
\hline Type I & Type III & Wild type & Negative & 1 & 128 & 0 & - & 0 & - & 1 & 128 \\
\hline Type I & Type IV & Wild type & Negative & 1 & $>2048$ & 0 & - & 0 & - & 1 & $>2048$ \\
\hline Type II & Type I & Wild type & Negative & 1 & $>2048$ & 0 & - & 0 & - & 1 & $>2048$ \\
\hline Type ॥ & Type I & Type VI & Negative & 0 & - & 0 & - & 1 & $>2048$ & 1 & $>2048$ \\
\hline Type III & Type I & Type II & Negative & 0 & - & 0 & - & 1 & 256 & 1 & 256 \\
\hline Type IV & Type V & Type I & Negative & 0 & - & 1 & 2048 & 0 & - & 1 & 2048 \\
\hline
\end{tabular}

${ }^{a}$ Type I: A279V; Type II: A297V/E225D; Type III: F267L/L281X; Type IV: G161R; Wild type: no mutations detected

b Type I: A434V; Type II: W147X; Type III: F1971; Type IV: A434V/G399S; Type V: C57X; Type VI: T313K; Wild type: no mutations detected

' Type I: G257D; Type II: D278E; Type III: deletion at position 717; Type IV: G322S; Type V: L162I; Type VI: E271Q; Type VII: G240R; Wild type: no mutations detected 
5 strains, the murA gene; 52 and 48 mutants, the $g l p T$ and $u h p T$ genes, respectively.

\section{Discussion}

A unique mechanism of action of fosfomycin made crossresistance to other antibiotic classes less common, which motivated physicians to reevaluate its ability to destroy drug-resistant pathogens, including MRSA [16]. In our study, elderly patients seemingly had the tendency of acquisition of fofsomycin-resistant MRSA infections, and the vast majority of those resistant strains were isolated from those hospitals located in central or southern Taiwan. The background mechanisms of this phenomenon need further investigation. Although the MRSA isolates exhibited high in vitro susceptibility to fosfomycin higher than $90 \%$, a significant increase in fosfomycin resistance rate during past decades (from $3.4 \%$ in 2002 to $11.0 \%$ in 2012) was observed in Taiwan. Among the fosfomycin-resistant isolates, a higher resistance rate to clindamycin, ciprofloxacin, erythromycin, and rifampicin was noted; however, trimethoprim/sulfamethoxazole and tetracycline displayed more favorable susceptibility. Type $\mathrm{I}_{u h p T}$ and type $\mathrm{I}_{g l p T}$ mutations predominantly caused fosfomycin resistance in our MRSA isolates, and the vast majority of isolates belonged to spa type t002.

Little was known about the MRSA fosfomycin resistance mechanism in epidemiological research, and very few literature reports were previously published $[15,28$, 29]. Of those studies, a large-scale surveillance conducted by $\mathrm{Fu}$ et al. [15] in China demonstrated a $13.4 \%$ (9/67) fosB-positive rate with two-thirds (6/9) belonging to ST5. A research study by Etienne et al. [28] revealed that 18 of $39(46.2 \%)$ S. aureus isolates, containing the fos $B$ gene, were highly resistant to fosfomycin, but only one MRSA isolate had the fosB gene (Zhejiang, China) [29]. In our present study, approximately one-fifth of the MRSA isolates with fosfomycin resistance carried the fos $B$ gene with the dominant t002 spa typing (11/12), which belonged to ST5. Our finding was similar to that described in Fu's report; it implicated the presence of clonal spread among the fos $B$-positive MRSA isolates, despite the previously reported triviality of fos $B$ [30].

MurA, a target enzyme involved in the biosynthesis of bacterial peptidoglycan, could be inactivated by fosfomycin via its binding to the active site of the enzyme [16]. However, mutations of the murA gene resulted in amino acid substitutions, rendering susceptible clinical isolates resistant to fosfomycin [16]. Fu et al. [15] illustrated that a murA mutation played an unclear role in the fosfomycin resistance in their study, and a type $\mathrm{II}_{\text {murA }}$ mutant was the most common among all murA mutations. Our results were different in this regard. The difference in the source of clinical specimens in these two studies might indicate that the mechanisms of fosfomycin resistance are different in Taiwan and mainland China.

The vast majority of the MRSA isolates in the present study possessed at least one of $g l p T$ and/or $u h p T$ mutations, implicating that the genes, encoding transporter mutants, contributed to fosfomycin resistance substantially. This result contrasted to the findings of the preceding study [15]. The prevailing subtype of mutations in the $g l p T$ and $u h p T$ genes was also different from that reported by $\mathrm{Fu}$ et al. [15]. Forty-eight fosfomycin-resistant MRSA isolates with dual resistance mechanisms ( $g l p T$ and $u h p T$ mutations) belonged to spa type t002, again implying clonal spread of fosfomycin-resistant MRSA.

The most prevalent and the second most common spa types were t002 and t037 in our study, respectively, revealing that fosfomycin resistance in MRSA isolates were correlated to some spa types. A similar correlation was noticed in other countries, including Sweden, Korea, China, Iran, Africa, Canada, and Brazil [31]. An international, or even intercontinental spread of specific fosfomycin-resistant MRSA clones may be occurring.

In the present study, the susceptibility of the MRSA isolates to various antibiotics was similar to that reported in the previous studies from Taiwan $[8,27]$ but different from that in other countries [32-34]. Variation in drug susceptibility between geographic areas might be due to the presence of different prevalent MRSA clones and the difference in antibiotic selective pressure.

The major limitation of the present study is that it was conducted using the clinical MRSA isolates in Taiwan; thus, worldwide generalization of the results should be made carefully.

\section{Conclusions}

In conclusion, our study illustrated that the fosfomycin resistance rate of the MRSA isolates increased significantly in the past, and mutations in the $g l p T$ and/or $u h p T$ genes were key for inducing fosfomycin resistance. These findings indicated to physicians that they should prescribe fosfomycin cautiously for treating MRSA infections empirically. Furthermore, t002 was the most frequently seen spa type among the fosfomycin-resistant MRSA isolates. This was comparable to that in other countries globally. Therefore, it is necessary to continuously monitor fosfomycin resistance and its mechanisms.

\section{Supplementary information}

Supplementary information accompanies this paper at https://doi.org/10. 1186/s13756-020-00790-x.

Additional file 1.

Abbreviations

MRSA: methicillin resistant Staphylococcus aureus; TSAR: Taiwan Surveillance of Antimicrobial Resistance; PCR: polymerase chain reaction; 
spa: Staphylococcal protein A; MDRO: multidrug-resistant organisms; XDRO: extensively drug-resistant organisms; VRE: vancomycin-resistant enterococci; ESBL: extended spectrum beta-lactamases; CLSI: Clinical and Laboratory Standards Institute

\section{Acknowledgements}

Not applicable.

\section{Authors' contributions}

YCL and PYC wrote the article and revised it critically for important intellectual content. JTY collected the data and did the analysis and interpretation of data. SCC was responsible for the conception and design of the study. SCC had given the final approval of the version to be published. All authors had read and approved the final manuscript.

\section{Funding}

There were no external or internal sources of specific funding for this paper, and the data were generated as part of the department's routine work.

\section{Availability of data and materials}

The datasets used during the current study are available from the corresponding author on reasonable request.

\section{Ethics approval and consent to participate}

The study was carried out in accordance with the principles stated in the Declaration of Helsinki, and approved by the Ethical Committee of National Taiwan University Hospital (NTUH-IRB No. 201504056RINB). The Review Board approved to waive inform consent due to the retrospective study design and the research posing no more than minimal risk.

\section{Consent for publication}

Not applicable.

\section{Competing interests}

The authors declared no conflict of interest.

\section{Author details}

'Department of Internal Medicine, Fu Jen Catholic University Hospital, Fu Jen Catholic University, New Taipei City, Taiwan. ${ }^{2}$ School of Medicine, College of Medicine, Fu Jen Catholic University, New Taipei City, Taiwan. ${ }^{3}$ Department of Internal Medicine, National Taiwan University Hospital, 7 Chung-Shan South Road, 100 Taipei, Taiwan. ${ }^{4}$ Institute of Infectious Diseases and Vaccinology, National Health Research Institutes, Tsu-Nan County, Taiwan.

Received: 8 February 2020 Accepted: 23 July 2020

Published online: 17 August 2020

\section{References}

1. Hendlin D, Stapley EO, Jackson M, Wallick H, Miller AK, Wolf FJ, et al Phosphonomycin, a new antibiotic produced by strains of streptomyces. Science. 1969;166:122-3.

2. Skarzynski T, Mistry A, Wonacott A, Hutchinson SE, Kelly VA, Duncan K. Structure of UDP-N-acetylglucosamine enolpyruvyl transferase, an enzyme essential for the synthesis of bacterial peptidoglycan, complexed with substrate UDP-N-acetylglucosamine and the drug fosfomycin. Structure. 1996:4:1465-74.

3. Pogue JM, Marchaim D, Abreu-Lanfranco O, Sunkara B, Mynatt RP, Zhao J, et al. Fosfomycin activity versus carbapenem-resistant Enterobacteriaceae and vancomycin-resistant Enterococcus, Detroit, 2008-10. J Antibiot (Tokyo). 2013;66:625-7.

4. Cho YH, Jung SI, Chung HS, Yu HS, Hwang EC, Kim SO, et al. Antimicrobial susceptibilities of extended-spectrum beta-lactamase-producing Escherichia coli and Klebsiella pneumoniae in health care-associated urinary tract infection: focus on susceptibility to fosfomycin. Int Urol Nephrol. 2015;47: 1059-66.

5. Tuon FF, Rocha JL, Formighieri MS, Sfair S, Bertoldi MB, Palmeiro JK, et al. Clinical Fosfomycin susceptibility of isolates with blaKPC-2 from Brazil. J Inf Secur. 2013;67:247-9.

6. Champion EA, Miller MB, Popowitch EB, Hobbs MM, Saiman L, Muhlebach MS, et al. Antimicrobial susceptibility and molecular typing of MRSA in cystic fibrosis. Pediatr Pulmonol. 2014;49:230-7.
7. Descourouez $J$, Jorgenson MR, Wergin JE, Rose WE. Fosfomycin synergy in vitro with amoxicillin, daptomycin, and linezolid against vancomycinresistant Enterococcus faecium from renal transplant patients with infected urinary stents. Antimicrob Agents Chemother. 2013;57:1518-20.

8. Lee YC, Chen PY, Wang JT, Chang SC. A study on combination of daptomycin with selected antimicrobial agents: in vitro synergistic effect of MIC value of $1 \mathrm{mg} / \mathrm{L}$ against MRSA strains. BMC Pharmacol Toxicol. 2019;20:25

9. Samonis G, Maraki S, Karageorgopoulos DE, Vouloumanou EK, Falagas ME. Synergy of fosfomycin with carbapenems, colistin, netilmicin, and tigecycline against multidrug-resistant Klebsiella pneumoniae, Escherichia coli, and Pseudomonas aeruginosa clinical isolates. Eur J Clin Microbiol Infect Dis. 2012;31:695-701.

10. Lowy FD. Staphylococcus aureus infections. N Engl J Med. 1998;339:520-32.

11. Rebiahi SA, Abdelouahid DE, Rahmoun M, Abdelali S, Azzaoui H. Emergence of vancomycin-resistant Staphylococcus aureus identified in the Tlemcen university hospital (north-West Algeria). Med Mal Infect. 2011;41:646-51.

12. Lingscheid T, Tobudic S, Poeppl W, Mitteregger D, Burgmann H. In vitro activity of doripenem plus fosfomycin against drug-resistant clinical blood isolates. Pharmacology. 2013;91:214-8.

13. Dinh A, Salomon J, Bru JP, Bernard L. Fosfomycin: efficacy against infections caused by multidrug-resistant bacteria. Scand J Infect Dis. 2012;44:182-9.

14. Florent A, Chichmanian RM, Cua E, Pulcini C. Adverse events associated with intravenous fosfomycin. Int J Antimicrob Agents. 2011;37:82-3.

15. Fu Z, Ma Y, Chen C, Guo Y, Hu F, Liu Y, et al. Prevalence of Fosfomycin resistance and mutations in murA, glpT, and uhpT in methicillin-resistant Staphylococcus aureus strains isolated from blood and cerebrospinal fluid samples. Front Microbiol. 2016;6:1544

16. Aghamali M, Sedighi M, Zahedi Bialvaei A, Mohammadzadeh N, Abbasian S, Ghafouri Z, et al. Fosfomycin: mechanisms and the increasing prevalence of resistance. J Med Microbiol. 2019;68:11-25.

17. Takahata S, Ida T, Hiraishi T, Sakakibara S, Maebashi K, Terada S, et al. Molecular mechanisms of fosfomycin resistance in clinical isolates of Escherichia coli. Int J Antimicrob Agents. 2010;35:333-7.

18. Nilsson Al, Berg OG, Aspevall O, Kahlmeter G, Andersson DI. Biological costs and mechanisms of fosfomycin resistance in Escherichia coli. Antimicrob Agents Chemother. 2003;47:2850-8.

19. Horii T, Kimura T, Sato K, Shibayama K, Ohta M. Emergence of fosfomycinresistant isolates of Shiga-like toxin-producing Escherichia coli O26. Antimicrob Agents Chemother. 1999;43:789-93.

20. Bernat BA, Laughlin LT, Armstrong RN. Fosfomycin resistance protein (FosA) is a manganese metalloglutathione transferase related to glyoxalase I and the extradiol dioxygenases. Biochemistry. 1997;36:3050-5.

21. Lee SY, Park YJ, Yu JK, Jung S, Kim Y, Jeong SH, et al. Prevalence of acquired fosfomycin resistance among extended-spectrum $\beta$-lactamase-producing Escherichia coli and Klebsiella pneumoniae clinical isolates in Korea and IS26-composite transposon surrounding fosA3. J Antimicrob Chemother. 2012;67:2843-7

22. Fillgrove KL, Pakhomova S, Schaab MR, Newcomer ME, Armstrong RN. Structure and mechanism of the genomically encoded fosfomycin resistance protein, Fos $X$, from listeria monocytogenes. Biochemistry. 2007:46:8110-20.

23. Sastry S, Doi Y. Fosfomycin: resurgence of an old companion. J Infect Chemother. 2016;22:273-80.

24. Clinical and Laboratory Standards Institute. Performance Standards for Antimicrobial Susceptibility Testing: Twenty-eighth Informational Supplement M100-S20. Wayne: CLSI; 2018.

25. Chen C, Xu X, Qu T, Yu Y, Ying C, Liu Q, et al. Prevalence of the fosfomycinresistance determinant, fosB3, in Enterococcus faecium clinical isolates from China. J Med Microbiol. 2014;63:1484-9.

26. Larsen AR, Stegger M, Sørum M. Spa typing directly from a mecA, spa and pvl multiplex PCR assay-a cost-effective improvement for methicillin-resistant Staphylococcus aureus surveillance. Clin Microbiol Infect. 2008:14:611-4.

27. Lee CY, Fang YP, Chang YF, Wu TH, Yang YY, Huang YC. Comparison of molecular epidemiology of bloodstream methicillin-resistant Staphylococcus aureus isolates between a new and an old hospital in Central Taiwan. Int J Infect Dis. 2019:79:162-8.

28. Etienne J, Gerbaud G, Fleurette J, Courvalin P. Characterization of staphylococcal plasmids hybridizing with the fosfomycin resistance gene fosB. FEMS Microbiol Lett. 1991;68:119-22. 
29. Wu D, Chen Y, Sun L, Qu T, Wang H, Yu Y. Prevalence of Fosfomycin resistance in methicillin-resistant Staphylococcus aureus isolated from patients in a University Hospital in China from 2013 to 2015. Jpn J Infect Dis. 2018;71:312-4.

30. Falagas ME, Athanasaki F, Voulgaris GL, Triarides NA, Vardakas KZ. Resistance to fosfomycin: mechanisms, frequency and clinical consequences. Int J Antimicrob Agents. 2019;53:22-8.

31. Asadollahi P, Farahani NN, Mirzaii M, Khoramrooz SS, van Belkum A, Asadollahi K, et al. Distribution of the Most prevalent Spa types among clinical isolates of methicillin-resistant and -susceptible Staphylococcus aureus around the world: a review. Front Microbiol. 2018;9:163.

32. Liang Y, Tu C, Tan C, El-Sayed Ahmed MAE, Dai M, Xia Y, et al. Antimicrobial resistance, virulence genes profiling and molecular relatedness of methicillin-resistant Staphylococcus aureus strains isolated from hospitalized patients in Guangdong Province, China. Infect Drug Resist. 2019:12:447-59.

33. Stefanaki C, leronymaki A, Matoula T, Caroni C, Polythodoraki E, Chryssou SE, et al. Six-Year Retrospective Review of Hospital Data on Antimicrobial Resistance Profile of Staphylococcus aureus Isolated from Skin Infections from a Single Institution in Greece. Antibiotics (Basel). 2017;6:39.

34. Peng H, Liu D, Ma Y, Gao W. Comparison of community- and healthcareassociated methicillin-resistant Staphylococcus aureus isolates at a Chinese tertiary hospital, 2012-2017. Sci Rep. 2018;8:17916.

\section{Publisher's Note}

Springer Nature remains neutral with regard to jurisdictional claims in published maps and institutional affiliations.

Ready to submit your research? Choose BMC and benefit from:

- fast, convenient online submission

- thorough peer review by experienced researchers in your field

- rapid publication on acceptance

- support for research data, including large and complex data types

- gold Open Access which fosters wider collaboration and increased citations

- maximum visibility for your research: over $100 \mathrm{M}$ website views per year

At $\mathrm{BMC}$, research is always in progress.

Learn more biomedcentral.com/submissions 